\title{
SPORTS ORIENTATION AND ITS ESSENCE IN CYCLING
}

\author{
Iv. Kolev* \\ Department „Technical and Ice Sports”, Coaches Faculty, National Sports Academy \\ „Vassil Levski“, Sofia, Bulgaria
}

\begin{abstract}
The development of sport results in cycling sports, as well as the enormous competition in the sport struggle for global hegemony in cycling disciplines, requires continuous improvement in the quality and effectiveness of the training process. The aim of the following theoretical study is to reveal the characteristic peculiarities and the essence of the sport orientation in the cycling sport.

Methods: The research was carried out using the following methods: literary sources study, document analysis, theoretical analysis and synthesis.

Results: Analysis. The modern development of cycling is characterized by the deepening of the knowledge of coaches and sport pedagogues in the field of sport orientation, with the creation of objective criteria for the latter, specific to each sport discipline. The choice of the type of sport that corresponds to the individual characteristics and abilities of the individual represents his/her sport orientation. It is primarily related to children's and adolescent sport. Good sport orientation increases the efficiency of sport selection.

Conclusion: Constantly increasing sport performance places ever-increasing demands on cyclists and, in turn, complicates the process of finding talents that combine qualities and abilities that determine success in cycling sports.
\end{abstract}

Key words: cycling, development, selection, description

\section{INTRODUCTION}

The development of sport results in cycling sports, as well as the enormous competition in the sport struggle for global hegemony in cycling disciplines requires continuous improvement in the quality and effectiveness of the training process. (1).

Cycling is a sport with a long history. This implies the accumulation of serious knowledge in the terms of the training process, sport selection of the most talented for practicing this sport, etc.

It is precisely the sports orientation that is the system of organizational and methodical activities, allowing to identify the directions of specialization in a certain variety of cycling disciplines (as road cycling, track cycling, mountain bike, BMX, etc.).

\footnotetext{
*Correspondence to: Ivan Kolev, National Sports Academy , Vassil Levski“", Coaches Faculty, Department ,, Technical and Ice Sports “ Sofia, Bulgaria,E-mail kolev_nsa@abv.bg
}

In order to reveal the essence, the peculiarities and the importance of the sport orientation, a necessary prerequisite is to clarify the relationship and the interaction between the selection and orientation, between sport selection and sport orientation. Both processes are indissolubly linked to one another; they are mutually determined and mutually posed. In general, this is a unified process of determining the correspondence between personality and activity, between individual and sport and sports discipline. This is an important starting point for the training of young cyclists and it is very important for the final result of the training.

The aim of the following theoretical study is to reveal the characteristic peculiarities and the essence of the sport orientation in the cycling sport.

\section{METHODS}

The research was carried out using the following methods: literary sources study, document analysis, theoretical analysis and synthesis. 
The subject of the following study is the system of selection and sport orientation of young cyclists in the Republic of Bulgaria.

The modern development of cycling is characterized by the deepening of the knowledge of coaches and sport pedagogues in the field of sport orientation, with the creation of objective criteria for the latter, specific to each sport discipline.

Sport orientation is inseparable from the sport selection. As a person's orientation to sport and sport activity, it is a basis on which to select bot the interests of sport and the interests of the individual, for the purposes of its upbringing and future (2).

In the sport orientation, the most suitable sport for the children to practice is chosen, the prospective directions of the motor activity in which it can reach mastery in sport. In a certain sport, the person must be able to reveal his/her abilities and potential. Good sport orientation enhances the quality and effectiveness of the sport selection that directs the child to sport. These are two different but interrelated activities - sport orientation and selection - the strategy and the tactics of a highly effective training process for achieving the desired goals and determining the most talented children from which an elite athlete can evolve. The problems of the sport orientation are related to solving the following research tasks: forming the ideal model, the one that will satisfy the high requirements of the given sport; forecasting - based on the scientific forecast to ensure effective selection of the individual athlete; organization of choice and selectin in a multi-annual aspect (3).

Initial selection in sport is one of the decisive factors for determining the genetic traits of children and their sport orientation (4).

The genetic polyformism (diversity) that determines the formation, development and manifestation of physical abilities is very necessary and useful. It is precisely for athletes, where the specific features of energy exchange in human body while performing physical work with different intensity and duration, that knowledge could help and regulate this process (5).

Some authors define the main task to be solved by the sport orientation of young cyclists as: "that the discovery of the relation between athlete's personality and sport discipline the revelation of significance, the value of correspondence simultaneously and interrelated for both the personality itself and the social group or society in general". Objective sport orientation is the process of the bilateral relationship between the system of organized and purposeful and external impact on one hand, and the personality as an object of impact on the other, and as a result, it makes a permanent, continuous and multilateral connection of the individual with sport activity and a specific discipline. It is characterized, above all, as being external to the personality, as an objective and outward-looking process directed to the personality. The existence of sport orientation is conditioned by the existence of a growing generation, of a person who has opportunities to be connected with sport (6). The same author states that there are two possibilities of contraction between the orientation towards sport success and the pursuit, the activity to achieve this success:

1. The athlete has a high level of focus on success and a low level of aspirations.

2. The athlete has a high level of aspirations with a low orientation towards success.

Sport selection, as a process of achieving a match between athlete and sport discipline, between personality and sport activity is actually unthinkable outside and regardless the process of sport orientation. Selection can only be done on individuals who have on or other direction and an inner orientation to practice particular activity. And because this orientation of the individual is done in order to achieve consistency between the individual activity, and the very correspondence between the individual and achieved activity, because the individual is directed towards the activity, both the process-sport selection, and the sport orientation are inseparable, connected to each other and are mutual conditions and posed. The scientific approach to the problems of sport selection, the relations of its essence and peculiarities cannot be truly completed without revealing the problems of the sport orientation (6).

In the sport orientation, the type of sport is chosen for the person and in the sport selection is chosen the specific person for the sport. Children's sport orientation and the display of their psycho-physical abilities influence on sport-pedagogical, psychosocial and psychopedagogical factors. Sports-pedagogical factors include: the training process with its structure, 
which is conditioned by the following components - the ration and interrelation of individual sides of the training process (physical, technical, psychic, theoretical, and tactical), stages and periods of multi-annual sports training; training means and methods; training loads and its adjustment in the training process (7).

Some authors define the sport orientation as a kind of social orientation. This is an activity to help children and young people choose their sport, according to their individual abilities, inclinations and interests. It is a system of organizational and methodical events that define the type of sport that corresponds to the individual characteristics of different persons (8).

Orientation solves the following tasks:

- Involves young people in systematic activities with physical culture and sports;

- Prepares children for elite sport in sports clubs;

- Organizes physical culture and sport in the educational institutions.

The sport orientation and selection of children for cycling is an important socio-pedagogical function. It consists in planning the physical activities of each adolescent according to his/her individual inclinations and natural abilities and the requirements of different cycling varieties (6). The same author also uses the notion of "sport orientation" - "a system of organizational and methodological events of a complex character, on the basis of which the individual's specialization in the defined sport is determined".

The choice of the type of sport that corresponds to the individual characteristics and abilities of the individual represent his/her sport orientation. It is primarily related to children's and adolescent sport. Good sport orientation increases the efficiency of sport selection.

The orientation towards the sport activity is the orientation of the personality, the interests of which to the development of the sport coincide with the public interests of different scales for increasing the sport mastery. Both sport and professional orientation are not and cannot be just a process of influence over personality, with the act of choosing a particular activity as a culmination.

In order to achieve high level sport results are required continuous and systematic work for a long period of time. All training and educational activities should be consistent and sustained.

\section{CONCLUSION}

The written above gave us reason to assume that sport orientation, in the current conditions of cycling sport development is of particular importance. Their solving on a scientific basis is an important prerequisite for enhancing the effectiveness of the multi-year training process. The current demand for forwardslooking for perspective athletes increases with each year. Success in orientation depends on the use of a comprehensive approach that allows a comprehensive assessment of potential prospect for future cyclists, taking into account social factors, pedagogical and biological index and the peculiarities of the psychological processes.

Current records in cycling are only available to be beaten by athletes with good health, special body composition, and high level of motor skills development, functional abilities and psychological features. Constantly increasing sport performance places ever-increasing demands on cyclists and, in turn, complicates the process of finding talents that combine qualities and abilities that determine success in cycling sports.

\section{REFRENCES}

1. Kolev, I. Basics of selection and orientation in cycling. It's a doct. NSA, Sofia. 2016.

2. Popov, N. Sports selection and orientation. S. 1976.

3. Dimitrova, B. Selection theory in sports gymnastics. Bolid-Ins., S.2014.

4. Hadzhiev, N., Dasheva, D. Selection and training in sports. SN, 1, 2009.

5. Akhmetov, I.I. Molecular Genetics of Sport: State and Pyrspectives. Teacherspsychological and biomedical problems of physical culture and sports, 5. 2007.

6. Filin, V.P., N.A. Fomin. The basics of youth sports. Fiss. M. 1980.

7. Mineva, M. Aerobic gymnastics, initial preparation. S. NSA Press. 2010.

8. Nikitushkin, V.G. Modern training of young athletes. M.2009. 